\title{
Expression of Metallothionein I/II and Ki-67 Antigen in Graves' Disease
}

\author{
PAWEL DOMOSLAWSKI ${ }^{1}$, BARTOSZ PULA ${ }^{2}$, MATEUSZ OLBROMSKI $^{2}$, BEATA WOJTCZAK $^{1}$, \\ TADEUSZ LUKIENCZUK ${ }^{1}$, MARZENNA PODHORSKA-OKOLOW ${ }^{2}$ and PIOTR DZIEGIEL ${ }^{2,3}$ \\ ${ }^{1}$ Department of General, Gastroenterological and Endocrine Surgery, \\ Medical University of Wroclaw, Wroclaw, Poland; \\ ${ }^{2}$ Department of Histology and Embryology, Medical University of Wroclaw, Wroclaw, Poland; \\ ${ }^{3}$ Department of Physiotherapy, Wroclaw University School of Physical Education, Wroclaw, Poland
}

\begin{abstract}
Background/Aim: The expression of metallothionein I/II (MT-I/II) was examined in thyroids of Graves' disease (GD) and nodular goiter $(N G)$ patients to determine its role as a potential marker of proliferation and autoimmune inflammation in the thyroid. Patients and Methods: MT-I/II and Ki-67 antigen expression was studied using immunohistochemistry in 72 GD and $24 N G$ patients. Results: MT-I/II expression was noted in the cytoplasm and nuclei of thyrocytes of GD and NG patients. Cytoplasmic and nuclear MT-I/II expression correlated strongly with $G D(r=0.51 ; p<0.0001)$ and $N G(r=0.50 ; p=0.0137)$. Cytoplasmic MT-I/II expression was significantly higher in GD (mean IRS 9.24 \pm 2.36 ) than in $N G$ (mean IRS 7.13 $\pm 2.51 ; p=0.0006)$ and correlated positively with Ki-67 antigen expression ( $r=0.28 ; p=0.0165)$. Nuclear MT-I/II expression was elevated in GD (mean 3.53 \pm 0.65 ) in comparison to $N G$ (mean $2.96 \pm 0.86 ; p=0.028$ ). Conclusion: MT-I/II may be a potential marker of GD in the thyroid and may be potentially involved in thyrocytes' proliferation.
\end{abstract}

Graves' Disease (GD) is an autoimmunological thyroid disease first described by Robert Graves in 1835 (1). Autoimmune diseases (AIDs) appear when the host immune system turns against its own antigens, leading to the dysfunction or destruction of target tissues and organs. AIDs may develop through different mechanisms involving

This study was supported by Grant No. ST-654 of the Wroclaw Medical University.

Correspondence to: Pawel Domoslawski, MD, Department of General, Gastroenterological and Endocrinological Surgery, Medical University of Wroclaw, Sklodowskiej-Curie 66, 50-369 Wroclaw Poland. Tel: +48 717842162, Fax: +48 717959619, e-mail: pawel.domoslawski@umed.wroc.pl

Key Words: Metallothionein, Ki-67, thyroid, Graves' disease, nodular goiter. immune deregulation, genetic predisposition and influence of environmental factors such as viral infections $(1,2)$. The disease appears in genetically susceptible patients, of which at greatest risk are patients with HLA DR3 (HLA DRB1*03) and DQA1*0501 alleles (1). Other identified risk factors include gender (women are up to 7-10 times more susceptible to GD than men), smoking, history of lithium treatment and low consumption of iodine (1). In GD, thyroid-reactive T-cells are generated and infiltrate the thyroid gland, leading to the production of B-cell derived TSH receptor (TSHR) directed antibodies. These in turn stimulate thyrocytes, resulting in their constant activation and the production of thyroid hormones, triiodothyronine (T3) and thyroxine (T4), that lead to hyperthyroidism (1, 3, 4). Antibodies directed against TSHR, also known as thyroid receptor antibodies (TRAb), are clinically classified into at least two groups: TSAb (thyroid stimulating antibodies) and TSBAb (thyroid stimulation-blocking antibodies). TSAb were shown to stimulate cAMP production and have been identified as the main cause of hyperthyroidism observed in the course of GD. On the contrary, TSBAb were shown to cause hypothyroidism, though in some cases they may possess some growth stimulating activity resulting in goiter formation (4). GD is also accompanied by an interstitial inflammation of the thyroid responsible for the generation of cytotoxic amounts of reactive oxygen species (ROS) (5-7).

Metallothionines (MT) are non-enzymatic, conservative among species, low-weight (6-7 kDA) metal-binding proteins, which were discovered by Margoshes and Vallee in 1957 in equine kidneys $(8,9)$. Their polypeptide chain is composed of 61-68 amino acids, out of which about $30 \%$ are cysteines (9). The thiol (-SH) groups of these proteins are responsible for metal binding (like $\mathrm{Zn}, \mathrm{Cd}, \mathrm{Cu}$ or $\mathrm{Hg}$ ) and transfer of $\mathrm{Cu}$ and $\mathrm{Zn}$ to the catalytic sites of various enzymes $(8,10)$. MT are encoded by a family of genes located in the human chromosome 16q13 (11). Four main MT isoform groups may be distinguished (MT-I, MT-II, MT- 
III and MT-IV) $(11,12)$. MT-I and MT-II in humans have at least eight functional isoforms (MT1A, MT1B, MT1E, MT1F, MT1G, MT1H, MT1X MT2A) and seven nonfunctional ones (MT1C, MT1D, MT1I, MT1J, MT1K, MT1L, MT2B) $(11,12)$. These isoforms are expressed in all tissues, whereas the expression of the other two functional isoforms is mainly found in the central nervous system (MTIII) and stratified squamous epithelium (MT-IV) $(13,14)$. However, numerous recent studies have shown that MT-III expression may also be noted in various malignancies and may be of significance with regard to patients' prognosis (15-20). MT-I/II expression may be found in the cytoplasm and nucleus of normal and malignant cells (21).

The binding of $\mathrm{Zn}$ and $\mathrm{Cu}$ ions by $\mathrm{MT}$ regulate their cellular levels and bioavailability to enzymes and transcription factors, which are involved in key cellular processes as DNA replication, transcription and translation $(8,22)$. In addition, they are capable of protecting the cells from harmful agents by forming inactive complexes with toxic metal ions or scavenging ROS $(23,24)$. MT-I/II expression was shown to be induced by several stress factors such as metal ions, ROS and glucocorticoids $(9,25-28)$. Lines of evidence suggest that MT-I/II may exert protective properties during inflammatory, regenerative as well as degenerative processes (10, 29-33). Moreover, cells with high mitotic rate are characterized by high expression of MT-I/II (9, 34-38). Indeed, MT-I/II overexpression was found in various types of human malignant tumors and often yielded prognostic and predictive significance $(34,39-42)$. Ki-67 is the most frequently used proliferation marker in routine histopathological diagnosis using paraffin sections $(36,38)$. It is a non-histone protein, expressed in the cell nucleus during proliferation, however its exact biological functions remain to be determined $(36,43)$. Numerous studies have shown a positive correlation between MT-I/II and Ki-67, which supports the proliferative role of MT-I/II (12, 35-38, 44).

Recently it has been suggested that MT-I/II may be a marker of innate immune system activation $(45,46)$. Taking into account that MT-I/II expression was studied in GD only on a limited number of cases and with no regard to its proliferative activity in thyrocytes of GD, we aimed at examining its expression in GD patients using immunohistochemical methods $(45,47)$. Moreover, MT-I/II expression was analyzed in regard to the intensity of the Ki-67 antigen expression in order to determine its possible impact on the proliferation of thyrocytes.

\section{Materials and Methods}

Tissue specimens. The study was performed on archival paraffinembedded thyroid samples of 72 patients with GD and 24 patients with nodular goiter (NG), which were operated in the Department of General, Gastroenterological and Endocrinological Surgery of the
Wroclaw Medical University between 2003 and2007. The mean age of patients with GD was $39.10 \pm 12.77$ (range $=16-68$ years), whereas NG patients mean age was 40.67 \pm 12.20 (range $=21-61$ years). Fifteen $(20.8 \%)$ men and $57(79.2 \%)$ women comprised the GD group, whereas five $(20.8 \%)$ men and $19(79.2 \%)$ women, the NG group. This study was approved by the Commission of Bioethics at the Wroclaw Medical University.

Histopathological examination and immunohistochemistry (IHC). Tissue samples were fixed in $10 \%$ buffered formalin, dehydrated and embedded in paraffin blocks. Renewed histopathological examination to verify the diagnosis was performed on $6-\mu \mathrm{m}$ thick sections stained with haematoxylin and eosin (H\&E). Immunohistochemical reactions were performed as previously described on 4- $\mu \mathrm{m}$ thick paraffin sections mounted on Superfrost Plus Slides (Menzel Gläser, Braunschweig, Germany), deparaffinised in xylene and gradually rehydrated (12). Briefly, in order to unmask the analyzed antigens, the sections were treated with Target Retrieval Solution buffer $\left(95^{\circ} \mathrm{C}, \mathrm{pH} 9,20 \mathrm{~min}\right)$ and subsequently cooled in distilled water and $1 \times \mathrm{PBS}$. Then, the blocking of endogenous peroxidase was performed by incubating the sections in $3 \%$ hydrogen peroxide for $5 \mathrm{~min}$. Next, the primary monoclonal antibodies directed against MT-I/II (clone E9, 1:100) and Ki-67 (clone MIB1, ready-to-use) were applied for $20 \mathrm{~min}$ at room temperature in an Autostainer Link 48. Visualisation of the studied antigens was performed with the use of biotinylated antibodies and streptavidin conjugated with horseradish peroxidase $\left(\right.$ EnVision $^{\mathrm{TM}}$ FLEX+ Mouse HRP). As a substrate for the reaction, 3,3'diaminobenzidine (DAB) was used. All the reactions were conducted using negative controls and all the slides were counterstained with haematoxylin. All the antibodies, reagents and equipment were obtained from DakoCytomation (Glostrup, Denmark).

Evaluation of IHC reactions. The IHC sections were evaluated by using a BX-41 light microscope (Olympus, Tokyo, Japan) by two independent pathologists who were blinded to the patients' clinical data. In doubtful cases a re-evaluation was performed using a doubleheaded microscope and the staining was discussed until a consensus was achieved. For the evaluation of the cytoplasmic MT-I/II expression, the immunoreactive score (IRS) of Remmele and Stegner was utilized (48). The scale takes into account the percentage of cells with positive reaction ( 0 points: absence of cells with positive reaction, 1 pt.: $1-10 \%$ cells, 2 pts.: $11-50 \%, 3$ pts.: $51-80 \%, 4$ pts.: over $80 \%$ cells with positive reaction) and the intensity of the colour reaction ( 0 : no reaction, 1: low intensity, 2: moderate intensity, 3 : intense colour), yielding a final score of 0 to 12 , which is the product of these two variables. Nuclear MT-I/II and Ki-67 antigen expression in follicular cells was assessed in whole tissue sections using a fivepoint cell positivity scoring system. The score was encoded as follows: 0 ( $0 \%$ cells stained), 1 (1-5\% cells stained), 2 (6-10\% cells stained), 3 (11-20\% cells stained), 4 (21-100\% cells stained) (49).

Statistical analysis. The obtained results were subjected to statistical analysis using Prism 5.0 (GraphPad, La Jolla, CA, USA). ShaphiroWilk normality test was utilized to test the normality of the distribution. The Mann-Whitney $U$-test was applied to compare the differences in expression of the studied markers between the groups. The correlation between the expression intensities of the selected markers was examined by using the Spearman's correlation test. The results were considered significant at $p<0.05$. 


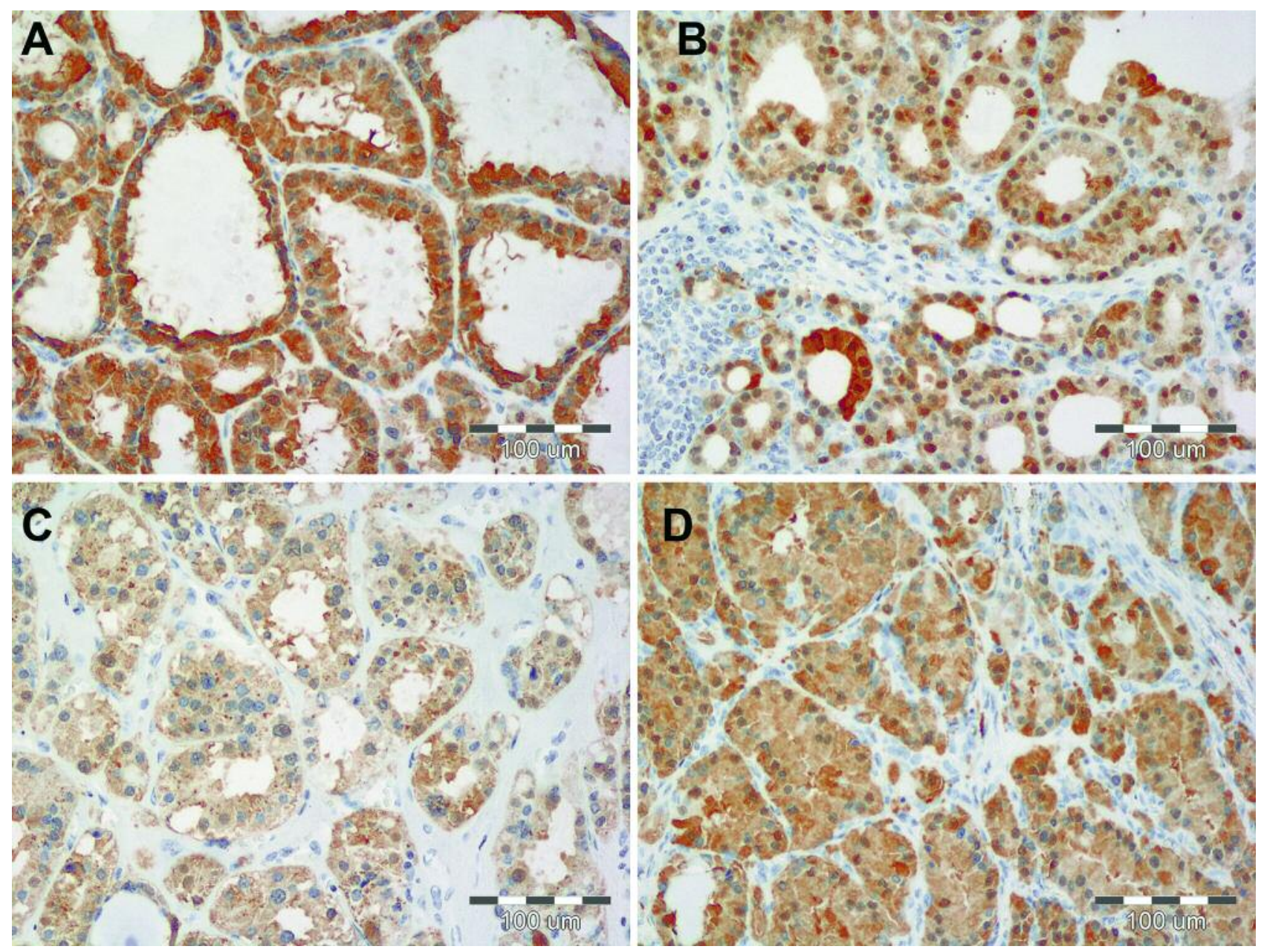

Figure 1. Comparison of cytoplasmic $(A, B)$ and nuclear $(C, D)$ MT-I/II expression in $N G(A, C)$ and $G D(B, D)$. Of note, the intensities of cytoplasmic, as well as nuclear reactions are weaker in NG tissues as compared to those observed in GD cases. In the latter a high concomitant intensity of expression of MT-I/II in the cytoplasm and nucleus of thyrocytes occurred.

\section{Results}

The expression of MT-I/II was noted in the cytoplasm, as well as in the nuclei of thyrocytes of GD and NG patients. It is worth notifying that GD cases were characterized by intense concomitant cytoplasmic and nuclear expression of MT-I/II (Figure 1). Both expression patterns (cytoplasmic and nuclear) of MT-I/II correlated strongly with GD $(\mathrm{r}=0.51$; $p<0.0001)$ and NG $(\mathrm{r}=0.50 ; p=0.0137)$ patients (Figure 2$)$. Statistical analysis revealed that the cytoplasmic MT-I/II expression was significantly higher in GD (mean IRS 9.24 \pm 2.36 ) than NG (mean IRS $7.13 \pm 2.51 ; p=0.0006$ ). Similarly, the nuclear MT-I/II expression was elevated in GD (mean 3.53 \pm 0.65 ) compared to NG (mean 2.96 \pm 0.86 ; $p=0.028$ ). Moreover, thyrocytes in GD showed a significantly higher Ki-67 antigen expression compared to
NG (mean $1.01 \pm 0.81 v s .0 .33 \pm 0.70 ; p<0.0001$; Figure 3). A significant positive correlation was observed between the cytoplasmic MT-I/II expression and Ki-67 antigen expression in GD ( $\mathrm{r}=0.28 ; p=0.0165$; Figure 4).

No significant correlations were noted between the cytoplasmic as well as nuclear MT-I/II expression patterns and patients' age in the particular study groups and when both groups were analyzed together. No differences in the expression intensities of the studied IHC markers in regard to patients' sex in GD and NG lesions were noted.

\section{Discussion}

In this research elevated cytoplasmic and nuclear expression of MT-I/II was shown in thyroids affected by GD in comparison to NG tissues. Our results are in accordance with 

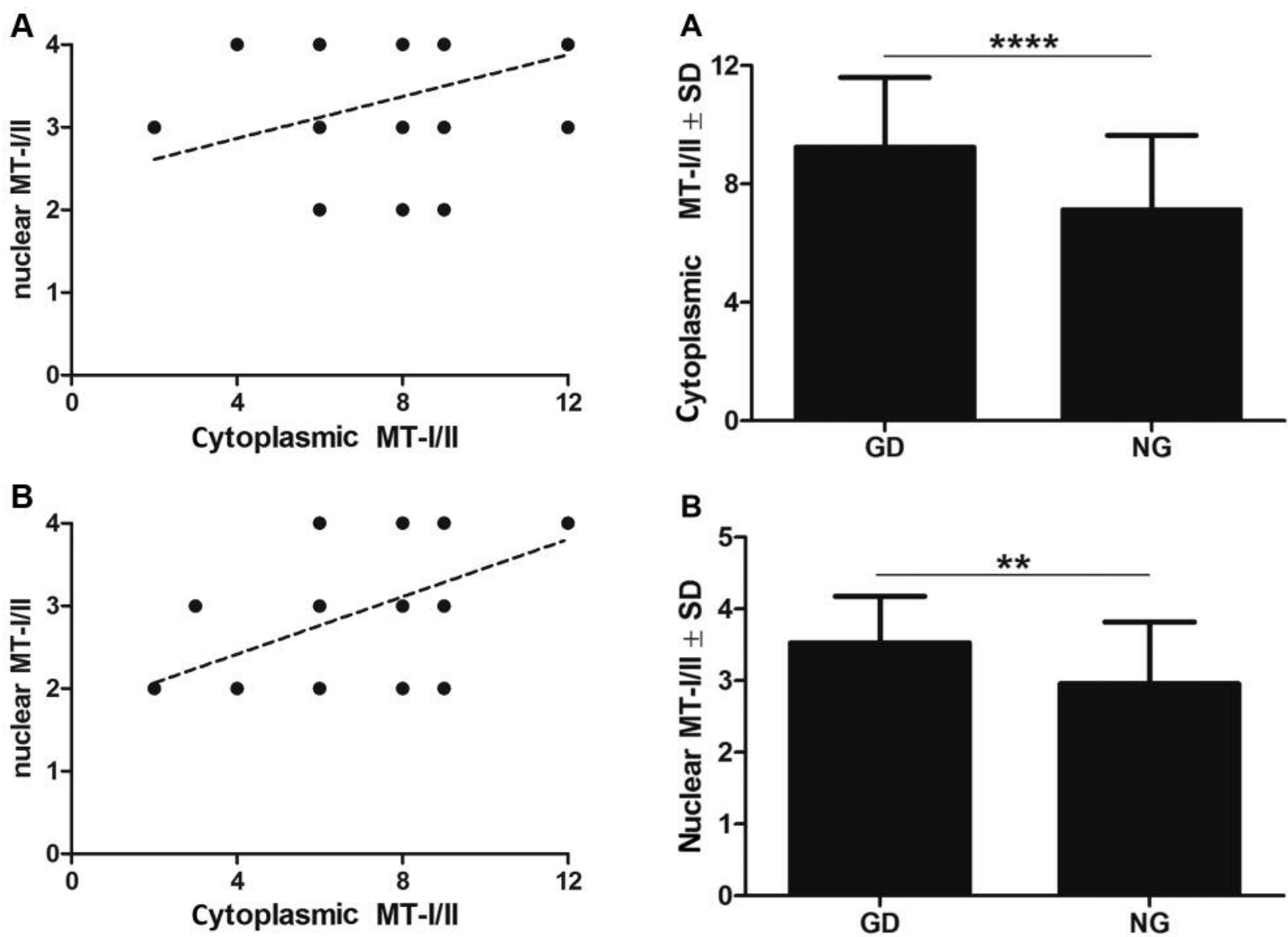

Figure 2. Spearman correlation test revealed a strong positive correlation of nuclear and cytoplasmic MT-I/II expression in GD $(r=0.51 ; p<0.0001 ; A)$ and $N G(r=0.50 ; p=0.0137 ; B)$.

the results of two previous studies on significantly smaller GD patient cohorts, which demonstrated an increased expression of MT-I/II in GD cases (45, 47). Moreover, our results also corroborate the results of the transcriptomic analysis, which revealed MT-I/II upregulation in comparison to normal thyroid glands (46).

In the descriptive study of Schmid et al., MT-I/II expression was noted in all 32 analyzed specimens of GD (47). Similar to our observations, the staining intensity, as well as the distribution of MT-I/II expression, differed among the analyzed cases. Moreover, MT-I/II staining was prominent in the hyperplastic areas of GD in contrast to other thyroid areas which lacked hyperplastic features (47). Interestingly, Ruiz-Riol et al. noted a strong cytoplasmic MT-I/II expression in 9 out of 14 studied cases of GD. In this study, none of the analyzed cases of Hashimoto's disease and NG was characterized by MT-I/II expression. Therefore MTI/II was proposed as a tissue marker of cellular stress in AID
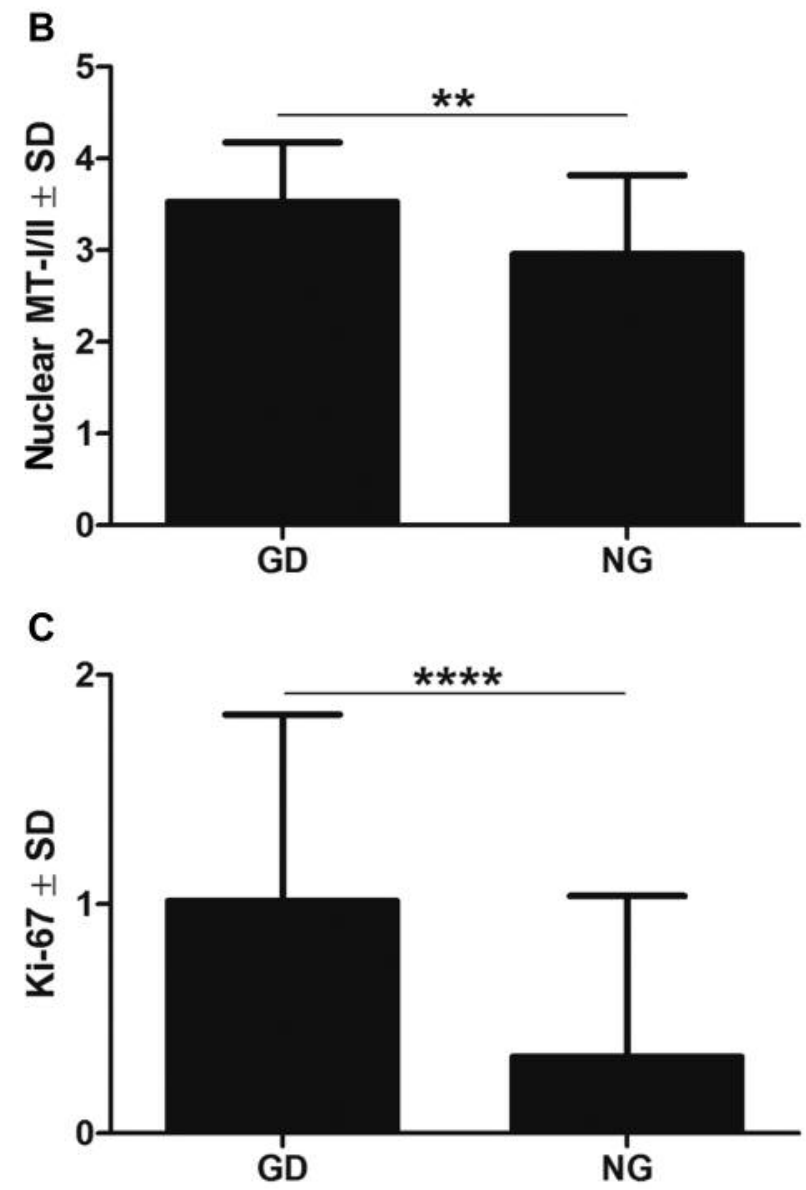

Figure 3. Cytoplasmic (A), nuclear (B) MT-I/II and Ki-67 antigen (C) expression in $G D$ and $N G$ patients. $* * p<0.01, * * * * p<0.0001$; MannWhitney test.

of the thyroid (45). Surprisingly, its expression increased in HT93 thyroid cell line upon combined stimulation with interferon $\gamma$ (IFN $\gamma$ ) and $\mathrm{ZnCl}_{2}$ with its peak expression achieved at $48 \mathrm{~h}$, whereas the HLA class II (regarded as a 


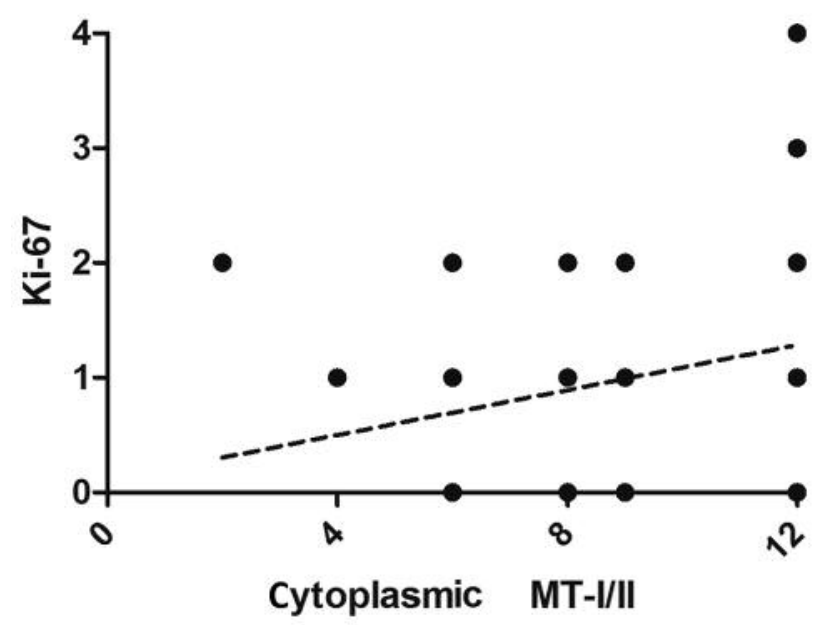

Figure 4. Correlation of cytoplasmic MT-I/II and Ki-67 antigen expression in GD patients ( $r=0.28 ; p=0.0165$; Spearman's correlation test).

marker of thyroid autoimmune inflammation) expression increased in response to IFN $\gamma$ alone (45). This points to a differentiated expression regulation of both proteins. Moreover, in IHC sections, thyrocytes with MT-I/II overexpression were characterized by a less prominent aberrant HLA class II expression (45).

Numerous studies have shown, that MT-I/II expression may be stimulated in response to several factors, e.g. ROS, glucocorticoids, metal ions or cytokines $(21,25,26)$. Cellular stress may also occur in the course of AID of the thyroid, including GD (50). Inflammatory reaction is induced by an autoimmune process leading to local generation of ROS in the thyroid (5). In normal, healthy conditions, ROS are formed as a metabolic product necessary for the cellular functions of thyrocytes. During the synthesis of thyroid hormones, hydrogen peroxide, a highly reactive oxidant, is formed, which together with oxidized iodine is used in the peroxidation reaction catalyzed by thyroid peroxidase. Peroxiredoxin, thioredoxin, glutathione peroxidase and catalase function as anti-oxidants protecting the thyrocytes from ROS damage (5). However, under pathological conditions, high levels of ROS may overcome the protective capacity of the aforementioned enzymes leading to "oxidative stress", resulting in the damage of thyrocytes $(5,51)$. In the thyroid, rapid changes in iodine intake may chemically stress the thyrocytes and trigger an autoimmune response $(5,45)$.

The observed elevated expression of MT-I/II in thyrocytes of GD could be, therefore, partially explained by the stimulatory role of generated ROS during the inflammatory process. However, it should be noted that although MT-I/II expression rose in vitro in HT93 in response to IFN $\gamma$ and $\mathrm{ZnCl}_{2}$ stimulation, no correlation was noted between MT-I/II expression and lymphocytic infiltration in GD tissues (45). Moreover, in light of the results of Ruiz-Riol et al., the mechanism of induction of MT-I/II expression seems to be different from that of HLA class II antigens, which are commonly expressed by thyrocytes affected by an autoimmune inflammatory reaction (45).

MT-I/II may act in thyrocytes affected by an inflammatory reaction as potent antioxidants reducing the damage caused by $\operatorname{ROS}(8,10)$. It was demonstrated that oxidative stress is capable of inducing the translocation of MT-I/II to the nucleus, where they exert their protective functions (52). Moreover, elevated expression of MT-I/II in GD thyrocytes may lead to the activation of particular transcription factors leading to increased cellular proliferation $(8,34,53)$. This hypothesis is strongly reflected by the results of this study, as MT-I/II expression correlated positively with the expression of the $\mathrm{Ki}-67$ antigen. Interestingly, these proliferative effects of MT-I/II seem to be restricted only to GD, as no correlation was observed between these proteins in follicular adenomas and cancers of the thyroid (54). Based on recent findings and the results obtained in this study, MTI/II in GD may be involved in diminishing the lesions caused during the interstitial inflammation $(10,33,45)$.

In summary, MT-I/II expression (cytoplasmic as well as nuclear) is elevated in GD compared to NG. Moreover, the importance of MT-I/II expression in GD is underlined by the observed positive correlation of its expression and the expression of the Ki-67 antigen. Although in light of the mentioned previously studies it is becoming apparent that MT-I/II may play a role in the pathogenesis of GD, its exact role remains to be determined.

\section{Acknowledgements}

This study was supported by Grant No. ST-654 of the Wroclaw Medical University.

The Authors thank Mrs Aleksandra Piotrowska, Mrs Bozena Przygodzka, Mrs Agnieszka Baranska and Mrs Elzbieta Polejko from the Department of Histology and Embryology of the Medical University of Wroclaw for their technical support.

\section{References}

1 Michels AW and Eisenbarth GS: Immunologic endocrine disorders. J Allergy Clin Immunol 125: S226-237, 2010.

2 Estienne V, Duthoit C, Reichert M, Praetor A, Carayon P, Hunziker W and Ruf J: Androgen-dependent expression of FcgammaRIIB2 by thyrocytes from patients with autoimmune Graves' disease: a possible molecular clue for sex dependence of autoimmune disease. FASEB J 16: 1087-1092, 2002.

3 Tomer Y, Ban Y, Concepcion E, Barbesino G, Villanueva R, Greenberg DA and Davies TF: Common and unique susceptibility loci in Graves and Hashimoto diseases: results of whole-genome screening in a data set of 102 multiplex families. Am J Hum Genet 73: 736-747, 2003. 
4 Ihara Y, Kanda Y, Seo M, Watanabe Y, Akamizu T and Tanaka Y: Growth stimulating antibody, as another predisposing factor of Graves' disease (GD): analysis using monoclonal TSH receptor antibodies derived from patients with GD. Endocr J 59: 571-577, 2012.

5 Zarkovic M: The role of oxidative stress on the pathogenesis of Graves' disease. J Thyroid Res 2012: 302537, 2012.

6 Poncin S, Van Eeckoudt S, Humblet K, Colin IM and Gerard AC: Oxidative stress: a required condition for thyroid cell proliferation. Am J Pathol 176: 1355-1363, 2010.

7 Poncin S, Colin IM, Decallonne B, Clinckspooor I, Many MC, Denef JF and Gerard AC: N-acetylcysteine and 15 deoxy\{delta\}12,14-prostaglandin $\mathrm{J} 2$ exert a protective effect against autoimmune thyroid destruction in vivo but not against interleukin-1 \{alpha\}/interferon \{gamma\}-induced inhibitory effects in thyrocytes in vitro. Am J Pathol 177: 219-228, 2010.

8 Nielsen AE, Bohr A and Penkowa M: The balance between life and death of cells: Roles of Metallothioneins. Biomark Insights 1: 99-111, 2007.

9 Dziegiel P: Expression of metallothioneins in tumor cells. Pol J Pathol 55: 3-12, 2004.

10 Pedersen MO, Larsen A, Stoltenberg M and Penkowa M: Cell death in the injured brain: roles of metallothioneins. Prog Histochem Cytochem 44: 1-27, 2009.

11 Thirumoorthy N, Shyam Sunder A, Manisenthil Kumar K, Senthil Kumar M, Ganesh G and Chatterjee M: A review of metallothionein isoforms and their role in pathophysiology. World J Surg Oncol 9: 54, 2011.

12 Werynska B, Pula B, Muszczynska-Bernhard B, Gomulkiewicz A, Piotrowska A, Prus R, Podhorska-Okolow M, Jankowska R and Dziegiel P: Metallothionein $1 \mathrm{~F}$ and $2 \mathrm{~A}$ overexpression predicts poor outcome of non-small cell lung cancer patients. Exp Mol Pathol 94: 301-308, 2013.

13 Uchida Y, Takio K, Titani K, Ihara Y and Tomonaga M: The growth inhibitory factor that is deficient in the Alzheimer's disease brain is a 68 amino acid metallothionein-like protein. Neuron 7: 337-347, 1991.

14 Quaife CJ, Findley SD, Erickson JC, Froelick GJ, Kelly EJ, Zambrowicz BP and Palmiter RD: Induction of a new metallothionein isoform (MT-IV) occurs during differentiation of stratified squamous epithelia. Biochemistry 33: 7250-7259, 1994.

15 Deng D, El-Rifai W, Ji J, Zhu B, Trampont P, Li J, Smith MF and Powel SM: Hypermethylation of metallothionein-3 CpG island in gastric carcinoma. Carcinogenesis 24: 25-29, 2003.

16 Werynska B, Pula B, Muszczynska-Bernhard B, Gomulkiewicz A, Jethon A, Podhorska-Okolow M, Jankowska R and Dziegiel $\mathrm{P}$ : Expression of metallothionein-III in patients with non-smallcell lung cancer. Anticancer Res 33: 965-974, 2013.

17 Dutta R, Sens DA, Somji S, Sens MA and Garrett SH: Metallothionein isoform 3 expression inhibits cell growth and increases drug resistance of PC-3 prostate cancer cells. Prostate 52: 89-97, 2002.

18 Garrett SH, Sens MA, Shukla D, Nestor S, Somji S, Todd JH and Sens DA: Metallothionein isoform 3 expression in the human prostate and cancer-derived cell lines. Prostate 41: 196202, 1999.

19 Sens MA, Somji S, Lamm DL, Garrett SH, Slovinsky F, Todd JH and Sens DA: Metallothionein isoform 3 as a potential biomarker for human bladder cancer. Environ Health Perspect 108: 413-418, 2000.
20 Kmiecik AM, Pula B, Suchanski J, Olbromski M, Gomulkiewicz A, Owczarek T, Kruczak A, Ambicka A, Rys J, Ugorski M, Podhorska-Okolow $\mathrm{M}$ and Dziegiel P: Metallothionein-3 increases triple-negative breast cancer cell invasiveness via induction of metalloproteinase expression. PLoS One 10: e0124865, 2015.

21 Pula B, Domoslawski P, Podhorska-Okolow M and Dziegiel P: Role of metallothioneins in benign and malignant thyroid lesions. Thyroid Res 5: 26, 2012.

22 Dziegiel P, Jelen M, Muszczynska B, Maciejczyk A, Szulc A, Podhorska-Okolow M, Cegielski $\mathrm{M}$ and Zabel M: Role of metallothionein expression in non-small cell lung carcinomas. Rocz Akad Med Bialymst 49: 43-45, 2004.

23 Gwinner W and Grone HJ: Role of reactive oxygen species in glomerulonephritis. Nephrol Dial Transplant 15: 1127-1132, 2000.

24 Kumari MV, Hiramatsu M and Ebadi M: Free radical scavenging actions of hippocampal metallothionein isoforms and of antimetallothioneins: an electron spin resonance spectroscopic study. Cell Mol Biol (Noisy-le-grand) 46: 627-636, 2000.

25 Hernandez J, Carrasco J, Belloso E, Giralt M, Bluethmann H, Kee Lee D, Andrews GK and Hidalgo J: Metallothionein induction by restraint stress: role of glucocorticoids and IL-6. Cytokine 12: 791-796, 2000.

26 Vasak M and Meloni G: Chemistry and biology of mammalian metallothioneins. J Biol Inorg Chem 16: 1067-1078, 2011.

27 Carrasco J, Hernandez J, Bluethmann $\mathrm{H}$ and Hidalgo J: Interleukin6 and tumor necrosis factor-alpha type 1 receptor deficient mice reveal a role of IL-6 and TNF-alpha on brain metallothionein-I and -III regulation. Brain Res Mol Brain Res 57: 221-234, 1998.

28 Gasull T, Giralt M, Hernandez J, Martinez P, Bremner I and Hidalgo J: Regulation of metallothionein concentrations in rat brain: effect of glucocorticoids, zinc, copper, and endotoxin. Am J Physiol 266: E760-767, 1994.

29 Campagne MV, Thibodeaux H, van Bruggen N, Cairns B and Lowe DG: Increased binding activity at an antioxidantresponsive element in the metallothionein-1 promoter and rapid induction of metallothionein-1 and -2 in response to cerebral ischemia and reperfusion. J Neurosci 20: 5200-5207, 2000.

30 Penkowa M, Moos T, Carrasco J, Hadberg H, Molinero A, Bluethmann $\mathrm{H}$ and Hidalgo $\mathrm{J}$ : Strongly compromised inflammatory response to brain injury in interleukin-6-deficient mice. Glia 25: 343-357, 1999.

31 Penkowa M, Giralt M, Moos T, Thomsen PS, Hernandez J and Hidalgo J: Impaired inflammatory response to glial cell death in genetically metallothionein-I- and -II-deficient mice. Exp Neurol 156: 149-164, 1999.

32 Penkowa M, Carrasco J, Giralt M, Moos T and Hidalgo J: CNS wound healing is severely depressed in metallothionein I- and II-deficient mice. J Neurosci 19: 2535-2545, 1999.

33 Chung RS, Hidalgo $J$ and West AK: New insight into the molecular pathways of metallothionein-mediated neuroprotection and regeneration. J Neurochem 104: 14-20, 2008.

34 Pedersen MO, Larsen A, Stoltenberg M and Penkowa M: The role of metallothionein in oncogenesis and cancer prognosis. Prog Histochem Cytochem 44: 29-64, 2009.

35 Werynska B, Pula B, Muszczynska-Bernhard B, Piotrowska A, Jethon A, Podhorska-Okolow M, Dziegiel P and Jankowska R: Correlation between expression of metallothionein and expression of Ki-67 and MCM-2 proliferation markers in nonsmall cell lung cancer. Anticancer Res 31: 2833-2839, 2011. 
36 Wojnar A, Pula B, Piotrowska A, Jethon A, Kujawa K, Kobierzycki C, Rys J, Podhorska-Okolow M and Dziegiel P: Correlation of intensity of MT-I/II expression with Ki-67 and MCM-2 proteins in invasive ductal breast carcinoma. Anticancer Res 31: 3027-3033, 2011.

37 Dziegiel P, Salwa-Zurawska W, Zurawski J, Wojnar A and Zabel M: Prognostic significance of augmented metallothionein (MT) expression correlated with $\mathrm{Ki}-67$ antigen expression in selected soft tissue sarcomas. Histol Histopathol 20: 83-89, 2005.

38 Gomulkiewicz A, Podhorska-Okolow M, Szulc R, Smorag Z, Wojnar A, Zabel M and Dziegiel P: Correlation between metallothionein (MT) expression and selected prognostic factors in ductal breast cancers. Folia Histochem Cytobiol 48: 242-248, 2010 .

39 Surowiak P, Materna V, Maciejczyk A, Pudelko M, Markwitz E, Spaczynski M, Dietel M, Zabel $M$ and Lage H: Nuclear metallothionein expression correlates with cisplatin resistance of ovarian cancer cells and poor clinical outcome. Virchows Arch 450: 279-285, 2007.

40 Surowiak P, Matkowski R, Materna V, Gyorffy B, Wojnar A, Pudelko M, Dziegiel P, Kornafel J and Zabel M: Elevated metallothionein (MT) expression in invasive ductal breast cancers predicts tamoxifen resistance. Histol Histopathol 20: 1037-1044, 2005.

41 Dziegiel P, Suder E, Surowiak P, Kornafel J and Zabel M: Expression of metallothionein in synovial sarcoma cells. Appl Immunohistochem Mol Morphol 10: 357-362, 2002.

42 Wojtczak B, Pula B, Gomulkiewicz A, Olbromski M, Podhorska-Okolow M, Domoslawski P, Bolanowski M, Daroszewski J and Dziegiel P: Metallothionein isoform expression in benign and malignant thyroid lesions. Anticancer Res 37: 5179-5185, 2017.

43 Wojnar A, Kobierzycki C, Krolicka A, Pula B, PodhorskaOkolow M and Dziegiel P: Correlation of Ki-67 and MCM-2 proliferative marker expression with grade of histological malignancy $(\mathrm{G})$ in ductal breast cancers. Folia Histochem Cytobiol 48: 442-446, 2010.

44 Szelachowska J, Dziegiel P, Jelen-Krzeszewska J, Jelen M, Tarkowski R, Wlodarska I, Spytkowska B, Gisterek I, Matkowski R and Kornafel J: Prognostic significance of nuclear and cytoplasmic expression of metallothioneins as related to proliferative activity in squamous cell carcinomas of oral cavity. Histol Histopathol 23: 843-851, 2008.

45 Ruiz-Riol M, Martinez-Arconada MJ, Alonso N, Soldevila B, Marchena D, Armengol MP, Sanmarti A, Pujol-Borrell R and Martinez-Caceres EM: Overexpression of metallothionein I/II: a new feature of thyroid follicular cells in Graves' disease. J Clin Endocrinol Metab 97: 446-454, 2012.
46 Ruiz-Riol M, Barnils Mdel P, Colobran Oriol R, Pla AS, Borras Serres FE, Lucas-Martin A, Martinez Caceres EM and PujolBorrell R: Analysis of the cumulative changes in Graves' disease thyroid glands points to IFN signature, plasmacytoid DCs and alternatively activated macrophages as chronicity determining factors. J Autoimmun 36: 189-200, 2011.

47 Schmid K, Greef M, Hittmair A, Tötsch M, Öfner D, DockhornDworniczak B, Böcker W and Jasani B: Metallothionein expression in normal, hyperplastic, and neoplastic thyroid follicular and parafollicular $\mathrm{C}$ cells using monoclonal anitmetallothionein antibody E9. Endocr Pathol 5: 114-122, 1994.

48 Remmele W and Stegner HE: [Recommendation for uniform definition of an immunoreactive score (IRS) for immunohistochemical estrogen receptor detection (ER-ICA) in breast cancer tissue]. Pathologe 8: 138-140, 1987.

49 Domoslawski P, Pula B, Lukienczuk T, Podhorska-Okolow M and Dziegiel P: Expression of estrogen and progesterone receptors and $\mathrm{Ki}-67$ antigen in Graves disease and nodular goiter. Folia Histochem Cytobiol 51: 135-140, 2013.

50 Abalovich M, Llesuy S, Gutierrez S and Repetto M: Peripheral parameters of oxidative stress in Graves' disease: the effects of methimazole and 131 iodine treatments. Clin Endocrinol (Oxf) 59: 321-327, 2003.

51 Sies H: Biological redox systems and oxidative stress. Cell Mol Life Sci 64: 2181-2188, 2007.

52 Takahashi Y, Ogra Y and Suzuki KT: Nuclear trafficking of metallothionein requires oxidation of a cytosolic partner. J Cell Physiol 202: 563-569, 2005.

53 Zeng J, Vallee BL and Kagi JH: Zinc transfer from transcription factor IIIA fingers to thionein clusters. Proc Natl Acad Sci USA 88: 9984-9988, 1991.

54 Krolicka A, Kobierzycki C, Pula B, Podhorska-Okolow M, Piotrowska A, Rzeszutko M, Rzeszutko W, Rabczynski J, Domoslawski P, Wojtczak B, Dawiskiba J and Dziegiel P: Comparison of metallothionein (MT) and Ki-67 antigen expression in benign and malignant thyroid tumours. Anticancer Res 30: 4945-4949, 2010
Received October 19, 2018

Revised November 5, 2018 Accepted November 6, 2018 